Document downloaded from:

http://hdl.handle.net/10251/54288

This paper must be cited as:

Barreiro, JJ.; Pons, A.; Barreiro, JC.; Castro-Palacio, JC.; Monsoriu Serra, JA. (2014). Diffraction by electronic components of everyday use. American Journal of Physics. 82(3):257-261. doi:10.1119/1.4830043.

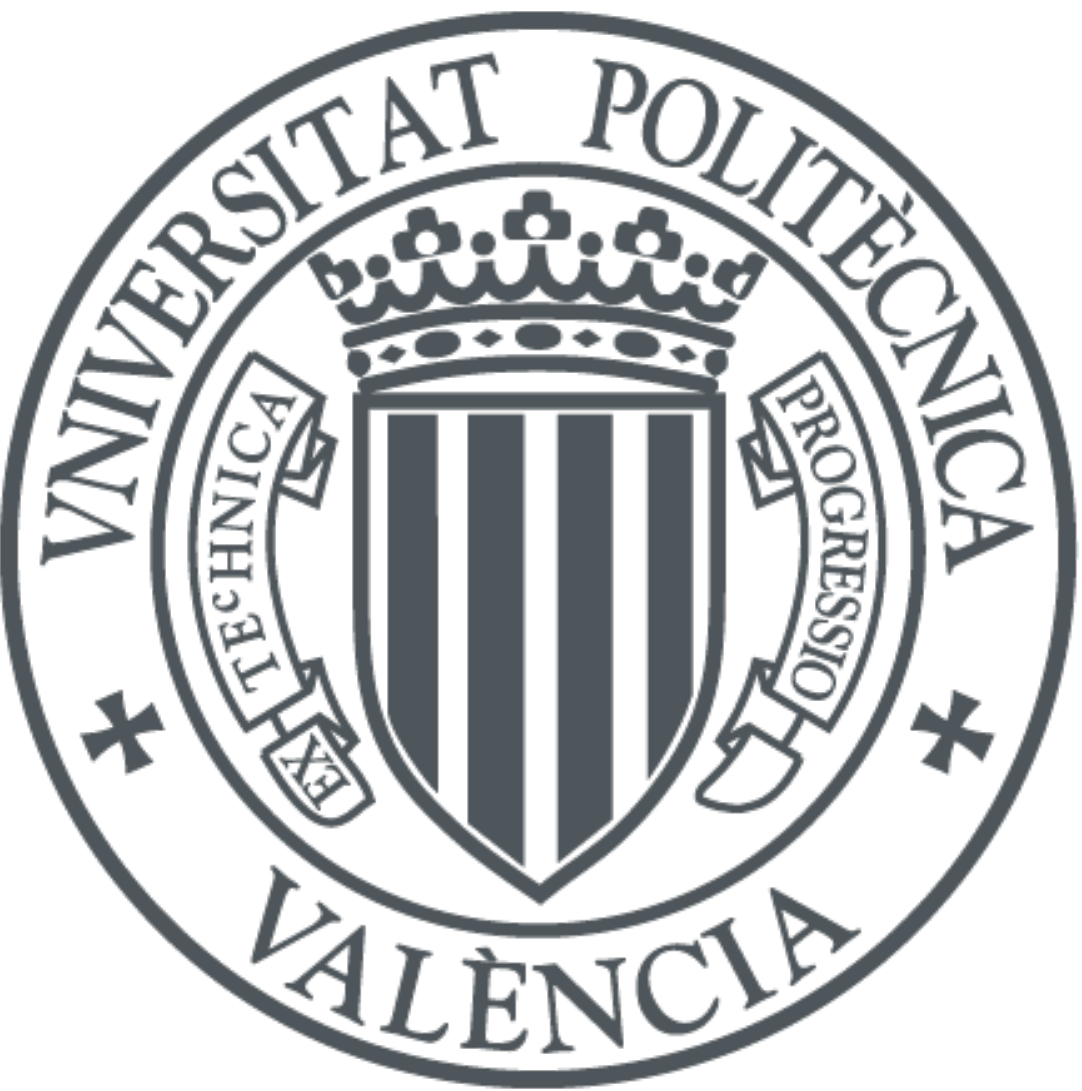

The final publication is available at

http://dx.doi.org/10.1119/1.4830043

Copyright American Association of Physics Teachers

Additional Information 


\title{
Diffraction by electronic components of everyday use
}

\author{
Jesús J. Barreiro \\ Departamento de Física Aplicada - IDF, \\ Universitat Politècnica de València, \\ Camí de Vera s/n, 46022, València, Spain. \\ Amparo Pons and Juan C. Barreiro \\ Departamento de Óptica, Universitat de València, 46100 Burjassot, València, Spain. \\ Juan C. Castro-Palacio \\ Departamento de Física, Universidad de Pinar del Río. Martí 270, \\ 20100, Pinar del Río, Cuba. and \\ Present affiliation: Department of Chemistry, \\ University of Basel. Klingelbergstr. 80, CH-4056, Basel, Switzerland. \\ Juan A. Monsoriu* \\ Centro de Tecnologías Físicas, Universitat Politècnica de València, \\ Cami de Vera s/n, 46022, Valencia, Spain.
}

(Dated: June 24, 2013)

\begin{abstract}
We propose the use of common electronics devices, such as CCD image sensors and LCD screens of mobile phones or digital cameras, to carry out simple optical experiments to study the phenomenon of diffraction. The proposed experiments can be performed as classroom demonstrations, or as laboratory experiments to study the spatial structure of these components by analyzing their diffraction patterns. This article presents the laboratory design and the experimental results obtained with these kinds of common electronic components.
\end{abstract}




\section{INTRODUCTION}

Most basic Physics courses for science or engineering students include the study of interference and diffraction as fundamental properties of all wave phenomena ${ }^{1,2}$. In the case of light, diffraction is observed when a light beam meets an obstacle or aperture (of size comparable to the light wavelength) that disturbs its propagation. Due to the wave behavior of light, the edges of the shadow produced by the aperture are not well defined, instead, interference stripes appear, that is, the diffraction phenomenon takes place at the edges of the aperture. This phenomenon plays an important role in the formation of images through real optical systems, since the small size of the lenses and the mirrors forming it limit the extension of the incident rays. This fact makes the image of a real point object not be a point but a diffraction spot whose dimensions limit the resolution of the imaging systems. This includes the diffraction effects caused by the pupil in the human eye.

Despite the undoubted importance that in many real systems the study of the diffraction of light waves has, its study is usually perceived by students as something of purely academic interest that requires of complicated mathematical treatment and very specific scientific components for observation, such as monochromatic light sources, apertures, slits of very small size or diffraction gratings. However, nothing is further from reality either in terms of interest or in the difficulty to make experiments.

In this respect, a great variety of creative works have been published concerning the wave phenomena of light ${ }^{3-7}$. For example, in reference ${ }^{8}$, diffraction experiments are done using slits made with graphic arts films. Compact discs (CD) as diffracting elements are studied in references ${ }^{9-12}$. The tracks of the CD represent a one-dimensional diffraction grating which can be used to perform low cost alternative experiments in Physics courses, which may be highly motivating for Electronics-related engineering students. Another example of construction of diffracting elements is the production of slits by a low cost photolithographic

process $^{13}$. In references ${ }^{14,15}$, the diffraction patterns of fractal Cantor set and Fibonacci sequence based gratings are studied, respectively. Typically, in many of these experiments a CCD image sensor is used for the digital registration of the diffraction patterns, with Ref. ${ }^{16}$ being one of the first didactic works on this topic. The cost of the equipment for this kind of experiment is usually high. This fact makes simulations of diffraction phenomena using new technologies a valuable alternative. In this respect, some appealing education software 
have been developed to study Young's double slit experiment and grating diffraction ${ }^{17}$.

Even though simulations are a good alternative to costly diffraction equipment, real laboratories are always most desired. In this work laboratory experiments related to the diffraction phenomenon using as diffracting gratings very common electronic components such as CCD (Charged-Coupled Device) image sensors ${ }^{18}$ or LCD (Liquid Crystal Display) screens ${ }^{19,20}$ are proposed. The experiments of this work contribute to the recycling of mobile phones and digital cameras by means of giving them a didactic use for basic Physics courses. As the light source, a monochromatic laser pointer is used. The analysis of the obtained diffraction patterns can be used to determine the characteristics of the pixel structure of these components and to determine the spatial resolution.

The outline of this paper is the following. In section II the basic theory of Fraunhofer diffraction is presented. In section III, the experimental set up is explained. Results for four diffracting objects (two CCD sensors and two LCD screens) are discussed in section IV and finally in section $\mathrm{V}$ some conclusions are drawn.

\section{BASIC THEORY}

First of all, let us consider the simple case of a vertical slit aperture of width $L$, illuminated perpendicularly with a parallel monochromatic light beam of wavelength $\lambda$ and irradiance $I_{0}$. The distribution of irradiance is obtained in the observation plane which is located at the distance $D$. This distance is large enough - in general- to consider the pattern as a Fraunhofer diffraction pattern. It can be demonstrated that for the considered aperture, the irradiance distribution on the observation screen is given by ${ }^{1,2}$ :

$$
I(x)=I_{0}\left[\frac{\sin (\pi L x / \lambda D)}{\pi L x / \lambda D}\right]^{2},
$$

where a small diffraction angle is assumed ( $D$ is large in comparison with $L$ ). The zeroirradiance positions in the diffraction pattern are given by

$$
x= \pm \lambda D / L, \pm 2 \lambda D / L, \pm 3 \lambda D / L, \ldots
$$

When the illuminated diffracting element is made of two identical apertures of width $L$ and separated by the distance $d$, two diffracted beams are generated, one coming from each 
aperture, which interfere with each other on a screen located at the distance $D$. In this case, the irradiance distribution on the observation screen can be expressed as:

$$
I(x)=4 I_{0}\left[\frac{\sin (\pi L x / \lambda D)}{\pi L x / \lambda D}\right]^{2} \cos ^{2}\left(\frac{\pi d x}{\lambda D}\right) .
$$

In the above expression, the interferential pattern, given by the term $\cos ^{2}(\pi d x / \lambda D)$, is modulated by the diffraction pattern of a single slit, - similar to the pattern given by equation 1 -, yielding finally the actual interference-diffraction pattern.

In the case of $N$ identical equi-spaced slits (1D diffraction grating), an interferencediffraction pattern made of a set of quasi-punctual maxima of irradiance (diffraction orders) aligned and equidistant between each other is obtained ${ }^{2}$. This pattern is given by the following equation,

$$
I(x)=I_{0}\left[\frac{\sin (\pi L x / \lambda D)}{\pi L x / \lambda D}\right]^{2}\left[\frac{\sin (N \pi d x / \lambda D)}{\pi d x / \lambda D}\right]^{2}
$$

It can be noticed that for $N=2$, the equation above transforms to equation 3. Again, the interference pattern produced by $N$ slits which is enveloped by the single slit pattern, is obtained. In this case, the main interference maxima are localized at,

$$
x=0, \pm \lambda D / d, \pm 2 \lambda D / d, \pm 3 \lambda D / d, \ldots
$$

So that the distance, $p$, between the consecutive diffraction orders depends on the distance $d$ between the slits of the grating according to the following expression:

$$
p=\frac{\lambda D}{d} .
$$

Finally, let us consider the more general case of an aperture which is replicated in two perpendicular directions $x$ and $y$ (2D diffraction grating). As in previous examples, it can be demonstrated that for this case, a bi-dimensional interference-diffraction pattern is obtained. It can be noticed that for each direction, $x$ or $y$, the distance between the maxima of irradiance (diffraction orders) depends on the period of the grating on the corresponding direction $\left(d_{x}\right.$ and $\left.d_{y}\right)$. In this respect, the following relations apply:

$$
p_{x}=\frac{\lambda D}{d_{x}}, \quad p_{y}=\frac{\lambda D}{d_{y}} .
$$


Similarly to the cases described above, the values of the irradiance corresponding to the maxima are also modulated by the diffraction pattern of the elemental aperture which is replicated. The diffraction orders of the grating do not have the same values of irradiance.

From all stated above, it can be concluded that from the analysis of the interferencediffraction patterns of a periodic object its spatial structure can be known. This idea will be used to determine the characteristics of the spatial structure of CCD image sensors and LCD screens. Both electronic components behave as 2D diffraction gratings since their pixel structure is formed by a large number of identical pixels (elemental apertures of a given form and size) which are replicated in two directions with a given period.

\section{EXPERIMENTAL SET UP}

The experimental set up used to obtain the diffraction patterns is very simple. As the light source, a monochromatic laser diode, Powerfix KH 4179, is used. The wavelength $\lambda$ $=650 \mathrm{~nm}$ provided by the manufacturer is verified using an Ocean Optics - HR4000 highresolution spectrometer to obtain a value of $(654 \pm 2) \mathrm{nm}$. The set up also includes a holder to fix the object that will be studied and a diffusing screen, on which, the diffraction pattern is observed.

One type of diffracting element to be used in our experiments is the LCD. It consists of a device whose functioning is based on the light modulating properties of liquid crystals ${ }^{18}$. LCDs are present in many everyday devices such as computer monitors, video game consoles, clocks, watches and calculators. When the diffracting element is an LCD screen, the diffraction pattern is obtained by transmission and is observed on the diffusing screen located a few meters away. Note that for a typical LCD whose distance between pixels is of the order of $200 \mu \mathrm{m}$, it implies that a laser of $2 \mathrm{~mm}$ of diameter covering 10 periods of the slit array is being used.

The other type of diffracting device used in our experiments is the CCD image sensor. CCDs image sensors are used for light detection in digital devices when high quality images are required, such as in digital cameras ${ }^{19}$. In a CCD image sensor, pixels are formed by p-doped MOSFET capacitors ${ }^{20}$. Unlike LCDs, the diffraction pattern of CCD sensors is

produced by reflection since it consists of opaque elements that do not allow the passage of the light through them. Besides, as the distance between pixels in a CCD is much smaller 
than for the LCD, typically of the order of $10 \mu \mathrm{m}$ or even smaller, the laser covers a region of 200 periods of the slit array on each direction.

The LCD and CCD have been obtained from obsolete digital cameras and mobile phones. The extraction of these electronic components is relatively simple, although in the case of the LCD there is a risk of breaking the screen, so that students should be careful. The results shown in the manuscript were obtained with LCD and CCD taken to the class and extracted by the students a few minutes before doing the experiments.

In order to determine the distance between the consecutive diffraction maxima ( $p_{x}$ on the $x$-axis direction and $p_{y}$ on the $y$-axis direction) in the diffraction patterns a vernier caliper is used. The distance $D$ between the diffracting element and the observation screen is determined with a measuring tape. By substituting these measurements in equation 7 , the period of the grating on each direction $\left(d_{x}\right.$ and $\left.d_{y}\right)$ can be determined. In order to verify the reliability of the results, the values of $d_{x}$ and $d_{y}$ derived from the diffraction pattern are compared with those measured directly with a calibrated TE 2000 microscope.

\section{RESULTS}

In the following, four examples of the results obtained with the aforementioned devices are presented, two for each, CCD image sensors (subsections A and B) and LCD screens (subsections $\mathrm{C}$ and $\mathrm{D}$ ).

\section{A. CCD image sensor of the Nokia 6102 mobile phone}

In table I, the experimental measurements of the distance between the consecutive diffraction maxima on the $x$ and $y$ axis of the diffraction pattern produced by the CCD sensor of the Nokia 6102 mobile phone (figure 2) are registered. The distance to the observation screen is $\mathrm{D}=(21.5 \pm 0.1) \mathrm{cm}$. The size of the pixel was calculated by taking the average values of the positions in the $x$ and $y$ axis. Comparative results between the measurements of the diffraction pattern and obtained with a microscope with a $10 \times$ objective are also shown in table I. In this table, $p_{\exp }$ represents the experimental values and $p_{\text {ave }}$ its average, $d_{D P}$ the size of the pixel calculated from the diffraction pattern (equation 7 ) and $d_{M}$ is same

quantity but obtained from the microscope. The error indicated for $p_{\exp }$ in the table is the 
TABLE I. Experimental values of the distance between the maxima in the diffraction pattern when the CCD image sensor of the phone Nokia 6102 is used.

\begin{tabular}{lcc}
\hline \hline Direction & $x$ & $y$ \\
\hline$p_{\exp }(m m)$ & $13.41 \pm 0.01$ & $13.31 \pm 0.01$ \\
& $13.46 \pm 0.01$ & $13.50 \pm 0.01$ \\
& $13.36 \pm 0.01$ & $13.41 \pm 0.01$ \\
$p_{\text {ave }}(m m)$ & $13.41 \pm 0.04$ & $13.40 \pm 0.08$ \\
\hline$d_{D P}(\mu m)$ & $10.49 \pm 0.11$ & $10.49 \pm 0.14$ \\
$d_{M}(\mu m)$ & $10.44 \pm 0.01$ & $10.44 \pm 0.01$ \\
\hline$D i s c(\%)$ & 0.12 & 0.12 \\
\hline \hline
\end{tabular}

caliper precision, $0.01 \mathrm{~mm}$. The error for the average over the measurements, $p_{\text {ave }}$, is the maximum value between the precision of the caliper, $0.01 \mathrm{~mm}$ and the standard deviation of the measurements. In tables from I to IV, the latter is larger than the precision, which is due to the lack of precision in localizing the maxima on the observation panel. In order to determine the error associated with the indirect measurement of $d_{D P}$ a standard procedure of propagation of errors has been used. Finally, the percentage discrepancy, Disc, between the pattern and microscope pixel sizes is shown in the last row of the table.

\section{B. CCD image sensor of the digital camera Canon Ixus 80 IS}

The experimental measurements for the diffraction pattern produced by the CCD sensor of the digital camera Canon Ixus 80 IS (figure 3), are registered in table II. In this case, pixels are smaller than in the previous example since this digital camera has a higher resolution. The distance to the observation screen is $\mathrm{D}=(21.5 \pm 0.1) \mathrm{cm}$. This table shows the same quantities as in table I. The image shown in Fig. 3c was, in this case, captured with a $40 \times$ microscope objective. 
TABLE II. Experimental values of the distance between the maxima in the diffraction pattern when the CCD image sensor of the digital camera Canon Ixus 80 IS is used.

\begin{tabular}{ccc}
\hline \hline Direction & $x$ & $y$ \\
\hline$p_{\exp }(m m)$ & $42.67 \pm 0.01$ & $42.61 \pm 0.01$ \\
& $42.60 \pm 0.01$ & $42.55 \pm 0.01$ \\
& $42.61 \pm 0.01$ & $42.61 \pm 0.01$ \\
$p_{\text {ave }}(m m)$ & $42.63 \pm 0.03$ & $42.59 \pm 0.03$ \\
\hline$d_{D P}(\mu m)$ & $3.30 \pm 0.03$ & $3.30 \pm 0.03$ \\
$d_{M}(\mu m)$ & $3.33 \pm 0.01$ & $3.33 \pm 0.01$ \\
\hline$D i s c(\%)$ & 0.23 & 0.23 \\
\hline \hline
\end{tabular}

\section{LCD screen of HTC Smartphone}

In table III, the experimental measurements for the diffraction pattern produced by the LCD screen of the HTC Smartphone (figure 4), are shown. The distance to the observation screen is $\mathrm{D}=(337.0 \pm 0.1) \mathrm{cm}$. Table III shows the same quantities as in tables I and II. The image shown in Fig. 4c was captured with the $10 \times$ microscope objective.

\section{LCD screen of a Sony DSC P73 digital camera}

The experimental measurements for the diffraction pattern produced by the LCD screen of a Sony DSC P73 digital camera (figure 5), are shown in table IV. The distance to the observation screen is $\mathrm{D}=(300.0 \pm 0.1) \mathrm{cm}$. This case is different from those presented before since pixels are arranged in a hexagonal lattice. Taking account the elementary reciprocal

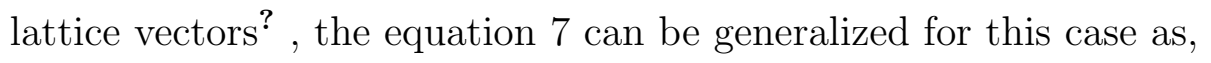

$$
p=\frac{\lambda D}{d \sin \alpha}
$$

where, $\alpha=60^{\circ}$, is the interior angle of the vertex of the triangle defined by three equivalent pixels (see Fig. $5 \mathrm{c}$ ), $d$ is the length of the triangle sides and $p$ is the distance between nearest neighbor diffraction orders (see Fig. 5 d). For the experimental values shown in 
TABLE III. Experimental values of the distance between the maxima in the diffraction pattern when the LCD screen of HTC Smartphone is used.

\begin{tabular}{ccc}
\hline \hline Direction & $x$ & $y$ \\
\hline$p_{\exp }(m m)$ & $12.55 \pm 0.01$ & $12.62 \pm 0.01$ \\
& $12.59 \pm 0.01$ & $12.56 \pm 0.01$ \\
& $12.67 \pm 0.01$ & $12.56 \pm 0.01$ \\
$p_{\text {ave }}(m m)$ & $12.6 \pm 0.05$ & $12.58 \pm 0.03$ \\
\hline$d_{D P}(\mu m)$ & $174.9 \pm 1.3$ & $175.2 \pm 1.0$ \\
$d_{M}(\mu m)$ & $175.23 \pm 0.01$ & $175.23 \pm 0.01$ \\
\hline Disc $(\%)$ & 0.05 & 0.004 \\
\hline \hline
\end{tabular}

TABLE IV. Experimental values of the distance between the maxima in the diffraction pattern when the LCD screen of a Sony DSC P73 digital camera is used.

\begin{tabular}{cc}
\hline \hline$p_{\exp }(m m)$ & $12.19 \pm 0.01$ \\
\hline & $12.15 \pm 0.01$ \\
$12.18 \pm 0.01$ \\
$p_{\text {ave }}(m m)$ & $12.17 \pm 0.02$ \\
\hline$d_{D P}(\mu m)$ & $186.2 \pm 0.9$ \\
$d_{M}(\mu m)$ & $183.94 \pm 0.01$ \\
\hline$D i s c(\%)$ & 0.31 \\
\hline \hline
\end{tabular}

table IV, the quantities are the same as in previous tables except that this time the size of the pixel, $d_{D P}$, is calculated from equation 8 . 


\section{CONCLUSIONS}

Simple devices for use in laboratory diffraction demonstrations are presented. Common electronic components, such as CCD image sensors, and LCDs recycled from mobile phones or digital cameras are used as diffracting apertures. Demonstrative classroom experiments, which can be also used as laboratory demonstrations, have been performed to determine the size of the pixels of different electronic components. The diffracting element of the electronic component is illuminated with a commercial laser pointer whose value of wavelength is verified with an optical spectrometer. The distance from the diffracting element to the observation screen is determined by using a measuring tape and the distances between maxima in the diffraction patterns with a vernier caliper. As can be seen, all components involved in the experimental set up are low cost. On the other hand, the use of electronic components of discarded mobiles and cameras directly contributes to the recycling of this type of materials.

Results obtained from the diffraction patterns are compared with those obtained using an optical microscope yielding a very good agreement (discrepancies lower than $0.5 \%$ ). Even when microscopes are not available, a further test in the case of the cameras can be done. The size of the pixels can be determined from the area of the screen divided by the number of pixels reported by the manufacturer which is usually expressed in megapixels. For example, in the case of the CCD of highest resolution (figure $3 \mathrm{~b}$ ), the value provided by the manufacturer is $8 \mathrm{Mp}$. The area of the CCD is $6.4 \times 4.7 \mathrm{~mm}^{2}$. Dividing the area by the distance between two equivalent pixels, $3.3 \mu \mathrm{m}$ (see table II), the quantity of pixels of the same type is $1940 \times 1424=2762560$ pixels $=2.76 \mathrm{Mp}$. Taking into account that there are three types of pixel (RGB), the resolution of the CCD, 8.3 Mp, is approximately the value reported by the manufacturer. The proposed experiments are simple and useful to complete the study of the interference and diffraction topics of light waves in basic Physics courses either at the qualitative or at the quantitative level.

\section{ACKNOWLEDGMENTS}

The authors would like to thank the financial support of the Ministerio de Economía y Competitividad (Projects: FIS2009-9135 and FIS2011-23175), the Generalitat Valenciana 
(Project: PROMETEO2009-077), Universitat Politècnica de València (PAID-05-11). This work has been developed by the teaching innovation groups GCID35/2009 and MoMa from the Universitat de València and Universitat Politècnica de València, respectively. We would also like to thank Dr. Michael Devereux for kindly revising the manuscript as a native English-speaking person.

*jmonsori@fis.upv.es

1 D. Halliday, R. Resnick, K. S. Krane, Physics, Volume 2 (John Wiley \& Sons, Inc., 2001).

2 E. Hecht, Optics (third edition) (Pearson, Addison-Wesley, United States, 2003).

3 T. Kr. Barik, A. Roy, and S. Kar, "A simple experiment on diffraction of light by interfering liquid surface waves," Am. J. Phys. 73, 725-729 (2005).

4 W. D. Furlan, G. Saavedra, and S. Granieri, "Simultaneous display of all the Fresnel diffraction patterns of one dimensional apertures," Am. J. Phys. 69, 799-802 (2001).

5 K. Wosilait, P. R. L. Heron, P. S. Shaffer, and L. C. McDermott, " Addressing student difficulties in applying a wave model to the interference and diffraction of light," Phys. Educ. Res., Am. J. Phys. Suppl. 67, S5-S15 (1999).

6 B. S. Ambrose, P. S. Shaffer, R. N. Steinberg, and L. C. McDermott, "An investigation of student understanding of single-slit diffraction and double-slit interference," Am. J. Phys. 67, 146-155 (1999).

7 K. K. Gan and A. T. Law, "Measuring slit width and separation in a diffraction experiment," Eur. J. Phys. 30, 1271-1276 (2009).

8 C. Lee, K. Shin, S. Lee, and J. Lee, "Fabrication of slits for Young's experiment using graphic arts films," Am. J. Phys. 78, 71-74 (2010).

9 P. M. Lane, R. V. Dommelen, and M. Cada, "Compact disc players in the laboratory: Experiments in optical storage, error correction, and optical fiber communication," IEEE Trans. Educ. 44, 47-60 (2001).

10 H. Kruglak, "The compact disc as a diffraction grating," Phys. Educ. 25, 255-256 (1990) .

11 J. E. Kettler, "The compact disc as a diffraction grating," Am. J. Phys. 59, 367-368 (1991).

12 C. Nöldeke, "Compact Disc Diffraction," Phys. Teach. 28, 484-485 (1990).

13 H. Slogoff, J. Mackowiak, M. Shishkov, and A. T. Johnson, "Photolithographic fabrication of 
diffraction and interference slit patterns for the undergraduate laboratory," Am. J. Phys. 72, 1328-1334 (2004).

14 J. A. Monsoriu, W. D. Furlan, A. Pons, J. C. Barreiro and M. H. Giménez, "Undergraduate experiment with fractal diffraction gratings," Eur. J. Phys. 32, 687-694 (2011).

15 M. J. McIrvin, "The Fibonacci ruler," Am. J. Phys. 61, 36-39 (1993).

16 C. de Izarra and O. Vallee, "On the use of linear CCD image sensors in optics experiments," Am. J. Phys. 62, 357-361 (1994).

17 J. Francés, M. Pérez-Molina, S. Bleda, E. Fernández, C. Neipp, and A. Beléndez, "Educational software for interference and optical diffraction analysis in Fresnel and Fraunhofer regions based on Matlab GUIs and the FDTD method," IEEE Trans. Educ. 55, 118-125 (2012).

18 D. J. R. Cristaldi, S. Pennisi, F. Pulvirenti, Liquid Crystal Display Drivers: Techniques and Circuits (Springer Science + Business Media B.V., Berlín, 2009).

19 J. R. Janesick, Scientific charge-coupled devices (SPIE Publications, Bellingham, Washington USA, 2001).

20 A. J. P. Theuwissen, Solid-State Imaging With Charge-Coupled Devices (Springer Science + Business Media B.V., Berlín, 1995). 


\section{FIGURES CAPTIONS}

a)

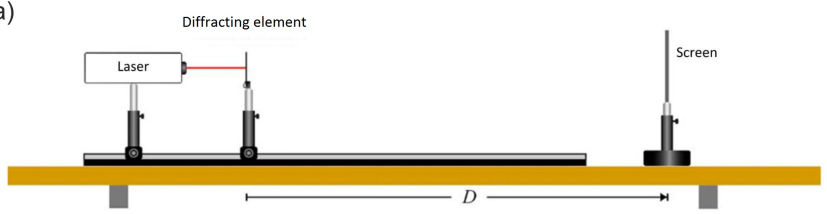

b)
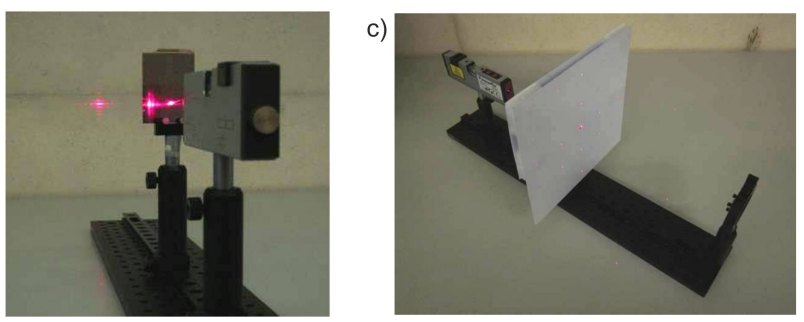

FIG. 1. (a) General schematic representation of the experimental set up, (b) device used to obtain the transmission diffraction pattern and (c) device used to obtain the reflection diffraction patterns.

a)

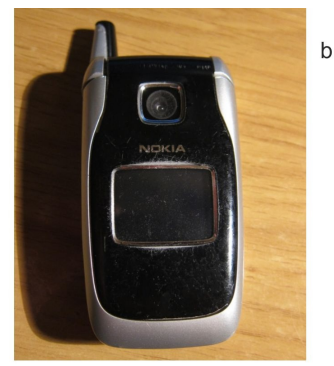

c)

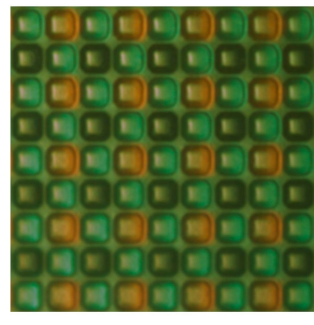

b)

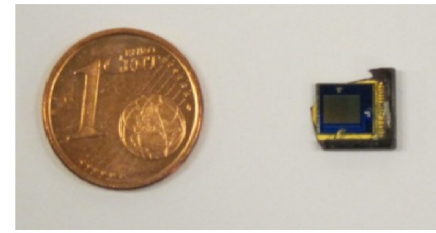

d)

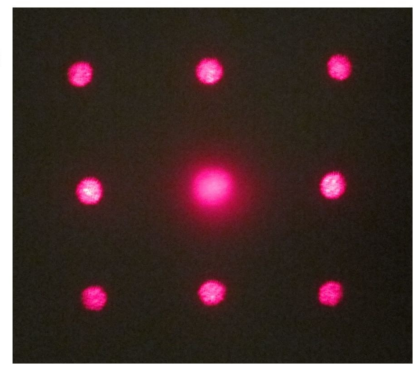

FIG. 2. (a) General view of the Nokia 6102 mobile phone, (b) photograph of the CCD sensor in comparison to a one-euro cent coin, (c) the microscope image of the sensor and (d) the central area of the reflection diffraction pattern. 


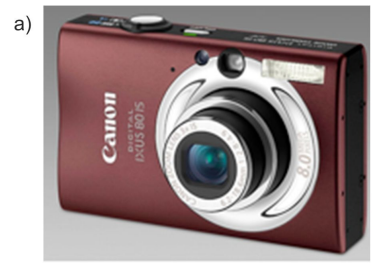

\section{b)}
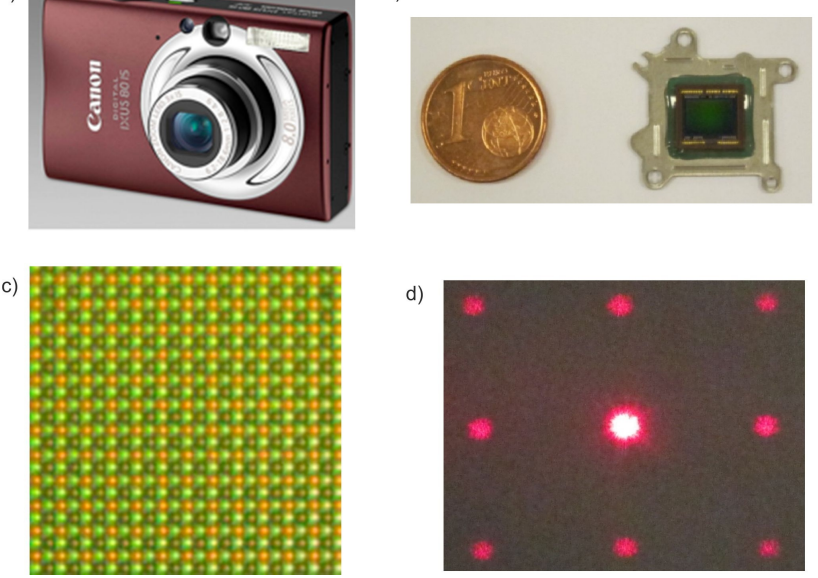

d)

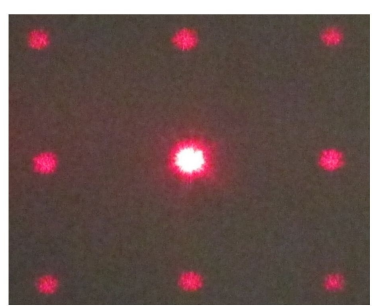

FIG. 3. (a) General view of the digital camera Canon Ixus 80 IS, (b) photograph of the CCD sensor in comparison to a one-euro cent coin, (c) the microscope image of the sensor and (d) the central area of the reflection diffraction pattern.

a)

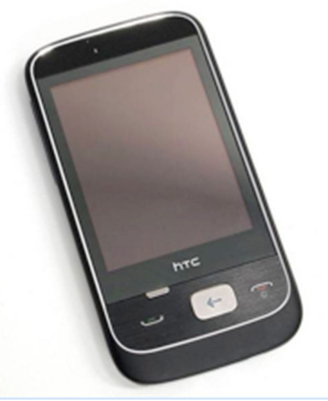

c)

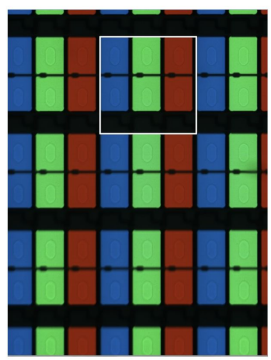

b)

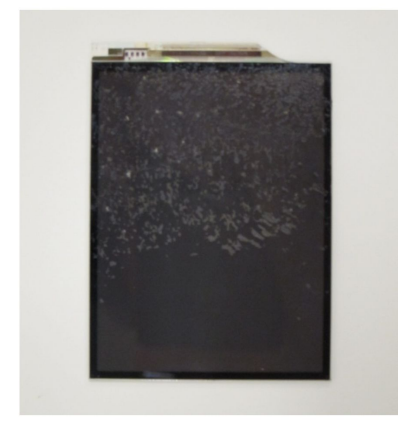

d)

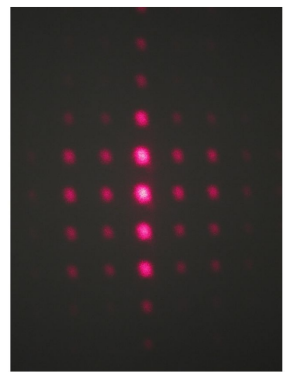

FIG. 4. (a) General view of the LCD screen of the HTC smartphone, (b) photograph of the screen panel, (c) its microscope image and (d) the central area of the transmission diffraction pattern. 

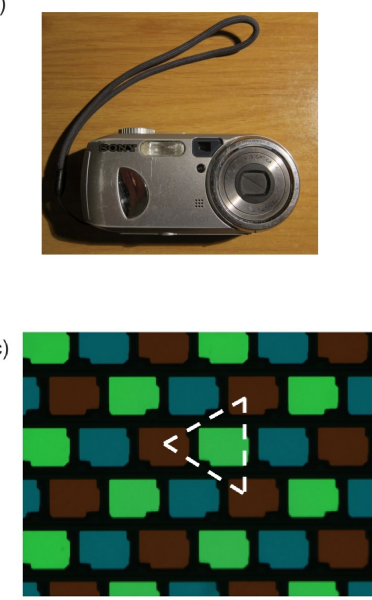
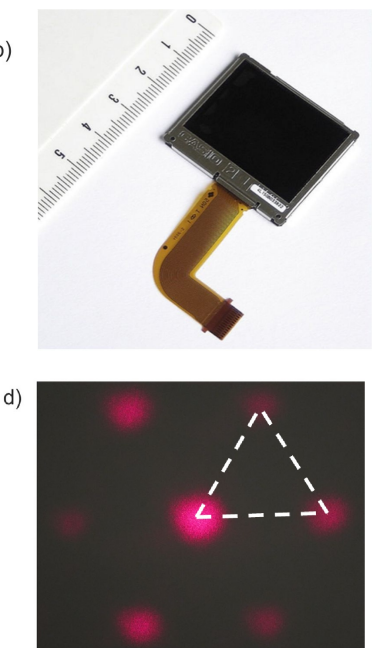

FIG. 5. (a) General view of the Sony DSC P73 digital camera, (b) photograph of the LCD screen, (c) its microscope image and (d) the central area of the transmission diffraction pattern. 